\title{
A dual function of SnRK2 kinases in the regulation of SnRK1 and plant growth
}

\author{
Borja Belda-Palazón $\mathbb{1}^{1,2,6}$, Mattia Adamo ${ }^{1,5,6}$, Concetta Valerio ${ }^{1,6}$, Liliana J. Ferreira', Ana Confraria1, \\ Diana Reis-Barata', Américo Rodrigues ${ }^{3,7}$, Christian Meyer ${ }^{4}$, Pedro L. Rodriguez ${ }^{2}{ }^{2}$ and \\ Elena Baena-González ${ }^{1}{ }^{凶}$
}

\begin{abstract}
Adverse environmental conditions trigger responses in plants that promote stress tolerance and survival at the expense of growth ${ }^{1}$. However, little is known of how stress signalling pathways interact with each other and with growth regulatory components to balance growth and stress responses. Here, we show that plant growth is largely regulated by the interplay between the evolutionarily conserved energy-sensing SNF1-related protein kinase 1 (SnRK1) protein kinase and the abscisic acid (ABA) phytohormone pathway. While SnRK2 kinases are main drivers of ABA-triggered stress responses, we uncover an unexpected growth-promoting function of these kinases in the absence of ABA as repressors of SnRK1. Sequestration of SnRK1 by SnRK2-containing complexes inhibits SnRK1 signalling, thereby allowing target of rapamycin (TOR) activity and growth under optimal conditions. On the other hand, these complexes are essential for releasing and activating SnRK1 in response to ABA, leading to the inhibition of TOR and growth under stress. This dual regulation of SnRK1 by SnRK2 kinases couples growth control with environmental factors typical for the terrestrial habitat and is likely to have been critical for the water-to-land transition of plants.
\end{abstract}

To cope with adverse environmental conditions, plants trigger cellular and whole-plant responses that confer protection but are often detrimental to growth ${ }^{1}$. Despite the negative impact of stress on crop productivity, how growth is modified by stress signalling pathways is poorly understood. One chief component of the stress response is SNF1-related protein kinase 1 (SnRK1), the plant ortholog of yeast sucrose non-fermenting 1 (SNF1) and mammalian AMP-activated protein kinase (AMPK), which drives vast metabolic and transcriptional readjustments that restore homeostasis and promote survival $^{2-4}$. Similar to SNF1 and AMPK, SnRK1 signalling is activated when energy levels decline during stress ${ }^{2}$, but is also induced by $\mathrm{ABA}^{5}$, a phytohormone essential for responses to stresses such as drought, extreme temperatures or salinity ${ }^{6}$. In the absence of ABA, type 2C phosphatases (PP2Cs) repress subgroup III SnRK2 kinases (SnRK2.2, SnRK2.3 and SnRK2.6 in Arabidopsis thaliana), keeping the pathway inactive ${ }^{7-11}$. Binding of ABA to its receptors enables PP2C sequestration and the release and activation of SnRK2s, which thereby induce protective responses and inhibit growth ${ }^{12,13}$.

Numerous studies have indicated cooperation between SnRK1 and ABA signalling in plant stress responses, growth and development ${ }^{5,14-22}$, but little is known of the underlying mechanisms. SnRK1 is a heterotrimeric complex and in Arabidopsis the $\alpha$-catalytic subunit is encoded by two genes, $S n R K 1 \alpha 1$ and

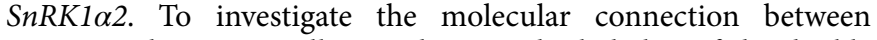
SnRK1 and ABA signalling and, given the lethality of the double snrk1 $\alpha 1$ snrk1 $\alpha 2$ knockout $^{2,23}$, we generated partial snrk $1 \alpha 1^{-1-}$ snrk $1 \alpha 2^{+/-}$loss-of-function mutants. These mutants show compromised SnRK1 accumulation (Supplementary Fig. 1) and signalling (Supplementary Fig. 2), as demonstrated by defective induction of SnRK1 marker genes in response to a transient dark treatment ${ }^{2}$. These are hereafter referred as sesquia2-1 or sesquia2-2 mutants, depending on the snrk $1 \alpha 2$ allele they harbour.

Despite being mostly similar to the wild-type (WT) during early development under normal conditions, sesquix 2 mutants fail to impose an ABA-dependent postgermination growth arrest ${ }^{24}$, developing green cotyledons in the presence of the hormone (Fig. 1a and Supplementary Fig. 3). Furthermore, sesqui 2 mutants are unable to reduce lateral root (LR) number in response to ABA to the same extent as control plants $(10,55$ and $41 \%$ of the mock

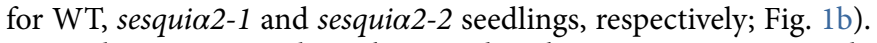
In similar assays, single snrk $1 \alpha 1$ and snrk $1 \alpha 2$ mutants are mostly indistinguishable from the WT, with only the snrk1 1 mutant being mildly defective in the repression of LR growth in response to ABA (Supplementary Fig. 4). Other ABA-regulated processes, such as germination (Supplementary Fig. 5a), primary root (PR) growth (Fig. 1b), transpiration rates (Supplementary Fig. 5b) and ABA marker gene induction (Supplementary Fig. 5c) appeared normal in sesquix 2 mutants, suggesting that the lack of SnRK1 affects only specific ABA responses and/or that SnRK1 signalling is not sufficiently compromised to visibly affect all ABA-related processes. The sesquia 2 mutants fail to repress LR growth also under low light conditions (Supplementary Fig. 6), showing that defective growth inhibition is not exclusive to ABA, and that, given the weak nature of this mutant, its defects are only apparent under conditions that substantially compromise growth in WT plants.

Given that all the observed ABA phenotypes of the SnRK1 sesquix 2 mutants relate to growth repression, and given the known antagonistic relationship between AMPK/SnRK1 and the growth-promoting target of rapamycin (TOR) kinase in animals ${ }^{25}$ and possibly in plants ${ }^{4}$, we examined the activation status of TOR in the sesquia 2-1 mutant in response to ABA. The phosphorylation of ribosomal protein S6 (RPS6 ${ }^{\mathrm{S} 240}$ ) in whole seedling extracts served

'Instituto Gulbenkian de Ciência, GREEN-IT Bioresources for Sustainability, Oeiras, Portugal. ${ }^{2}$ Instituto de Biología Molecular y Celular de Plantas, Consejo Superior de Investigaciones Científicas-Universidad Politécnica de Valencia, Valencia, Spain. ${ }^{3}$ MARE Marine and Environmental Sciences Centre, ESTM, Instituto Politécnico de Leiria, Peniche, Portugal. ${ }^{4}$ Institut Jean-Pierre Bourgin (IJPB), INRAE, AgroParisTech, Université Paris-Saclay, Versailles, France. ${ }^{5}$ Present address: BPMP, University of Montpellier, CNRS, INRA, Montpellier SupAgro, Montpellier, France. ${ }^{6}$ These authors contributed equally: Borja Belda-Palazón, Mattia Adamo, Concetta Valerio. ${ }^{7}$ Deceased: Américo Rodrigues. ${ }^{\circledR}$ e-mail: ebaena@igc.gulbenkian.pt 


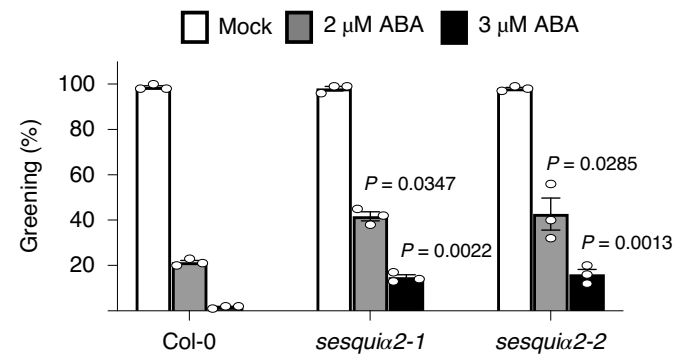

b

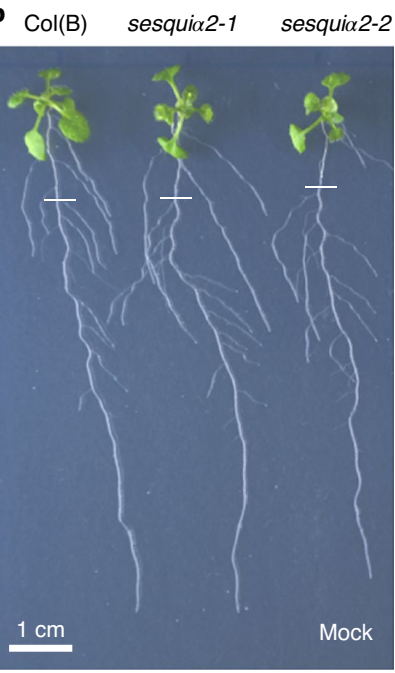

Col(B) sesquix2-1 sesquix2-2

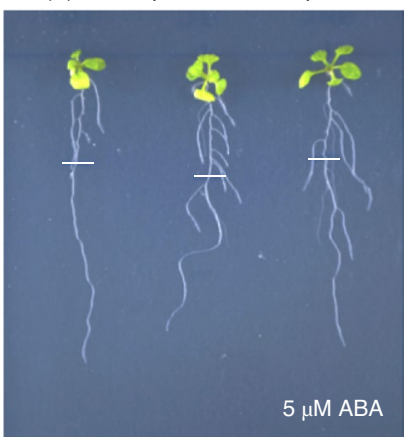

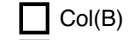

$\square$ sesquix2-1
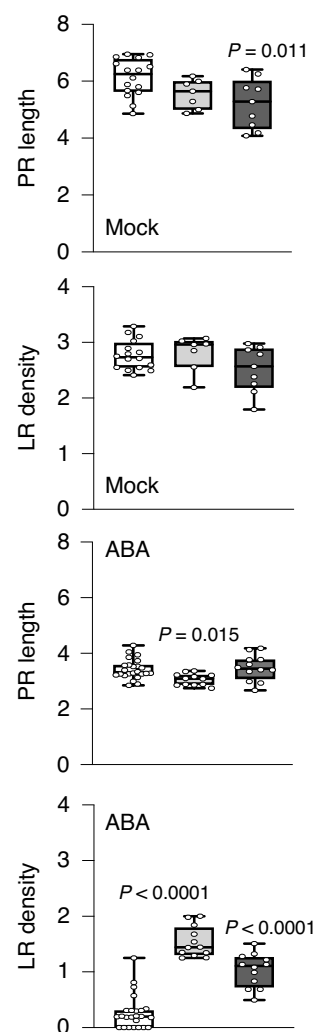

sesquia 2-2

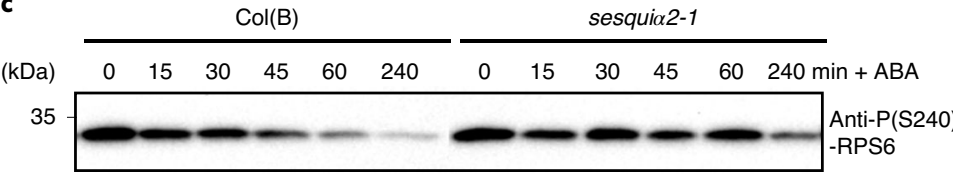

35

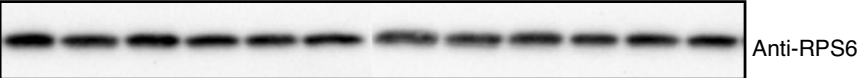

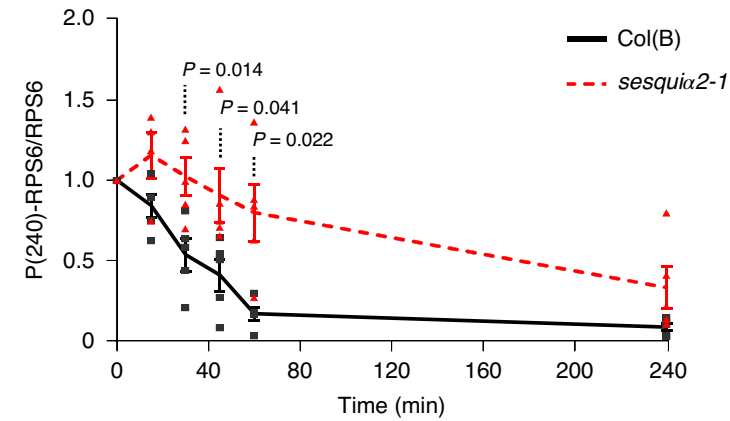
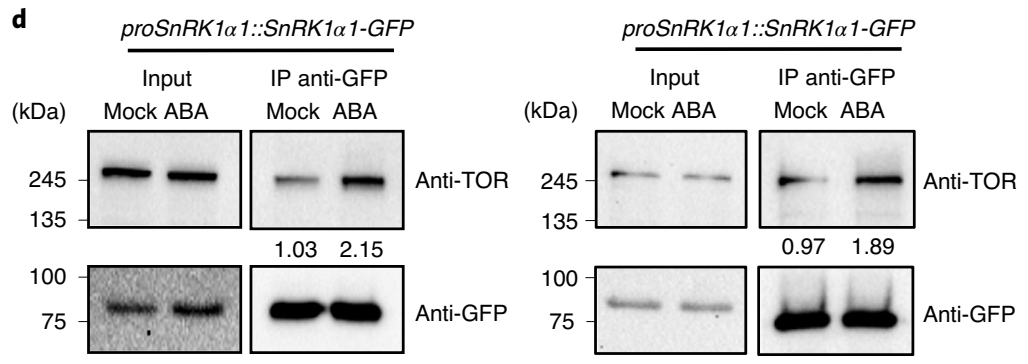

e

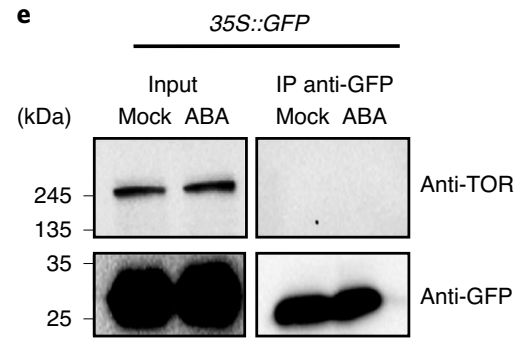

f

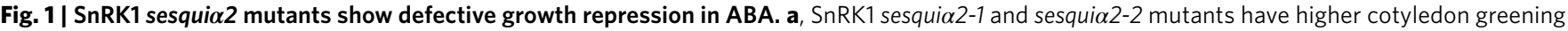
rates than control plants in ABA. Graph shows the percentage of green and expanded cotyledons in seedlings grown for $15 \mathrm{~d}$ on $0.5 \times \mathrm{MS}$ with or without $\mathrm{ABA}(n=3,100$ seeds per genotype each experiment; error bars indicate s.e.m.). $P$ values denote statistically significant differences for comparisons to the Col-0 control (one-way analysis of variance (ANOVA) with a Tukey honestly significant difference (HSD) test). b, SnRK1 sesquia2-1 and sesquia2-2 mutants have higher LR density than control plants in ABA. Left panels, representative pictures of seedlings grown vertically on $0.5 \times$ MS medium with BASTA for $5 \mathrm{~d}$ and transferred to $0.5 \times \mathrm{MS}$ with or without ABA for $8 \mathrm{~d}$. Right panels, quantification of PR length and LR density from six independent experiments (total number of plates: WT mock, $n=16$; sesqui $2-1$ mock, $n=7$; sesqui $\alpha 2-2$ mock, $n=9$; WT ABA, $n=24$; sesqui $2-1$, ABA $n=12$; sesquia2-2 mock, $n=12$. Total number of seedlings: 36-72 per genotype and condition). Upper and lower box boundaries represent the first and third quantiles, respectively, horizontal lines mark the median and whiskers mark the highest and lowest values. $P$ values denote statistically significant differences for comparisons to control plants (one-way ANOVA with Tukey HSD test). Col(B), BASTA-resistant Col-0 expressing 35S::GFP, was used as a control. Scale bar, $1 \mathrm{~cm}$. c, Repression of TOR signalling in response to ABA is slower in SnRK1 sesquix2-1 mutants than in Col(B) control plants. Seedlings were treated with $50 \mu \mathrm{M}$ ABA for the indicated times and TOR activity was subsequently analysed from total protein extracts using immunoblotting and RPS6 ${ }^{5240}$ phosphorylation as readout. Graph corresponds to the average of five independent experiments (error bars indicate s.e.m.). $P$ values denote statistically significant differences (two-tailed Welch $t$-test). All samples were run in the same gel but images were cropped for showing first the Col(B) series. d, TOR interacts with SnRK1 $\alpha 1$, and the interaction is enhanced twofold in ABA. Fourteen-d-old seedlings expressing SnRK1 $\alpha 1-G F P$ were treated with mock or $50 \mu \mathrm{M}$ ABA for 40 min, GFP-tagged proteins were immunoprecipitated from total protein extracts and coimmunoprecipitation of TOR was assessed by immunodetection with TOR specific antibodies. Two independent experiments are shown. Numbers refer to the relative intensity of the corresponding TOR band. e,f, TOR is not coimmunoprecipitated with GFP alone (e) or with SnRK2.2-GFP (f). Fourteen-d-old seedlings expressing 35S::GFP or proSnRK2.2::SnRK2.2-GFP were treated and analysed as in d. Two independent experiments were performed with similar results $(\mathbf{e}, \mathbf{f})$. 
as a faithful readout ${ }^{26}$, confirming previous results on the inhibition of TOR signalling by ABA and its dependency on SnRK2 kinases ${ }^{27}$ (Supplementary Fig. 7). In response to ABA, the sesquia2-1 mutant showed a slower inhibition of TOR along all the analysed $4 \mathrm{~h}$ time-course sampling points (Fig. 1c), indicating that SnRK1 $\alpha 1$ is required for repressing TOR activity in response to ABA. To assess if the SnRK $1 \alpha$ effect is direct, we next analysed the physical interaction between SnRK $1 \alpha 1$ and TOR by coimmunoprecipitation (co-IP), using a green fluorescent protein- (GFP-)tagged SnRK1 $\alpha 1$ line $^{14}$, a 35S::GFP control line, and antibodies recognizing TOR or its regulatory protein RAPTOR. In whole seedling extracts TOR was readily coimmunoprecipitated with SnRK1 $\alpha 1-$ GFP (Fig. 1d) but not with GFP alone (Fig. 1e). A basal SnRK1 $\alpha 1-$ TOR interaction was detected in mock conditions, and it was enhanced twofold by a short ABA treatment $(40 \mathrm{~min}$, Fig. 1d). Similar results were obtained for RAPTOR (Supplementary Fig. 8a,b), confirming previous observations that SnRK1 $\alpha 1$ and RAPTOR interact in planta ${ }^{4,28}$. These results were further corroborated for the endogenous proteins using TOR immunoprecipitation and immunodetection of SnRK1 $\alpha 1$ (Supplementary Fig. 8d). A recent study demonstrated that the repression of TOR by ABA is SnRK2-dependent ${ }^{27}$. However, using a GFP-tagged SnRK2.2 line ${ }^{29}$ we were unable to detect any interaction of TOR or RAPTOR with SnRK2.2-GFP either in mock- or ABA-treated plants (Fig. If and Supplementary Fig. 8c). Furthermore, none of the three SnRK2s (SnRK2.2/2.3/2.6) could be detected in immunoprecipitates of endogenous TOR in either of the two conditions (Supplementary Fig. 8d), altogether suggesting that, despite being necessary for repressing TOR in response to $\mathrm{ABA}^{27}$, SnRK2s may not be directly involved in TOR repression and that TOR is instead inhibited by SnRK1.

To explore the molecular connection between SnRK2 and SnRK1, we first examined their potential colocalization. As previously reported, SnRK1 $\alpha 1$ and SnRK2.2 were prominently expressed in the root tip, in LR primordia and in subsequent stages of LR development (Supplementary Fig. 9) ${ }^{14,29}$. At the subcellular level, both kinases were present in the cytosol and the nucleus, being particularly enriched in the latter (Supplementary Fig. 9). To investigate the SnRK1-SnRK2 physical interaction we next performed reciprocal co-IP experiments using the same material as for the microscopy analyses (roots). In mock-treated seedlings we retrieved a clear interaction between SnRK1 $\alpha 1$ and SnRK2 in both directions (Fig. 2a,b), while neither SnRK2 nor SnRK1 $\alpha 1$ could be detected in immunoprecipitates of GFP alone (Supplementary Fig. 10a). The reported interaction of both SnRK2 (refs. ${ }^{9,10}$ ) and SnRK1 $\alpha 1$ (ref. ${ }^{5}$ ) with clade A PP2C phosphatases served as positive controls (Fig. 2c,d). Treatment with $\mathrm{ABA}$ caused a marked reduction in all three interactions (Fig. 2a-d, for the PP2CA interactions please note that this is relative to the total PP2CA amount, which is known to be strongly increased by ABA through transcriptional activation ${ }^{30}$ ), suggesting that the three proteins may be part of the same complexes. A similar effect of $A B A$ on the SnRK2-SnRK1 $\alpha 1$ interaction was observed using the same material and conditions as for evaluating the interaction with TOR (whole seedlings, 40 min ABA treatment; Supplementary Fig. 10b,c), showing the interaction is rapidly reduced by the hormone. Using seedlings overexpressing FLAG-tagged SnRK2.3 and SnRK2.6 we could further demonstrate that the interaction between SnRK1 $\alpha 1$ and SnRK2s as well as the reduction of this interaction by ABA is shared by all three ABA-induced SnRK2 kinases (Supplementary Fig. 10d,e).

To assess whether the interaction between SnRK1 and SnRK2 is direct or whether it is dependent on the presence of PP2Cs we used bimolecular fluorescence complementation assays in Nicotiana benthamiana (Fig. 2e and Supplementary Fig. 11a,b). Expression of $\mathrm{YFP}^{\mathrm{N}}-\mathrm{SnRK} 1 \alpha 1$ with $\mathrm{YFP}^{\mathrm{C}}-\mathrm{SnRK} 2 \mathrm{~s}$ and a nuclear targeted red fluorescent protein (RFP) control (mRFP-NLS) did not result in yellow fluorescent protein (YFP) reconstitution (Fig. 2e and Supplementary Fig. 11a,b). However, coexpression of the two kinases with PP2CA-RFP yielded a very strong YFP signal in the nucleus, indicating that the presence of PP2CA enables SnRK2s to interact with SnRK1 $\alpha 1$. Moreover, a kinase dead SnRK2.6 variant $\left(\text { SnRK2.6 } 6^{\mathrm{G} 33 \mathrm{R}}\right)^{31}$ was also able to interact with SnRK1 $\alpha 1$ in a PP2CA-dependent manner, demonstrating that the SnRK1 $\alpha 1-S n R K 2$ interaction does not rely on the kinase activity of the latter (Supplementary Fig. 11a,b). Immunoblot analyses of the infiltrated leaf sectors confirmed the expression of $\mathrm{YFP}^{\mathrm{N}}-\mathrm{SnRK} 1 \alpha 1$ and $\mathrm{YFP}^{\mathrm{C}}-\mathrm{SnRK} 2 \mathrm{~s}$ in all samples (Supplementary Fig. 11c).

To investigate the relationship between SnRK1 and SnRK2 kinases we crossed the snrk1 1 single mutant to the snrk2.2/2.3 double mutant (hereafter referred as snrk2d) to assess their genetic interaction (Supplementary Fig. 12). We reasoned that, given the partial impairment of $\mathrm{ABA}$ responses in this mutant ${ }^{7}$ (as opposed to the full impairment of the snrk2.2/2.3/2.6 mutant (snrk2t) ${ }^{32-34}$ ), a potential contribution from the snrkl 1 1 mutation could be more easily detected in this background. Despite having mostly no effect on its own (Supplementary Fig. 4), the snrk1 1 1 mutation clearly enhanced the ABA insensitivity of the snrk2d mutant, increasing its germination and cotyledon greening rates (Fig. 3a,b), and the formation of LRs in ABA (Fig. 3c). This indicates that the SnRK1 pathway contributes to specific ABA signalling outputs. Furthermore, the sensitization of the snrk1 1 mutation by the snrk2d background in ABA, indicates that SnRK2s may promote SnRK1 signalling in these conditions. To investigate whether SnRK2s can phosphorylate and activate SnRK1 directly, we first immunoprecipitated active and inactive HA-tagged SnRK2.3 variants expressed in Arabidopsis mesophyll protoplasts treated under mock or ABA conditions. Selective activation of SnRK2.3 by ABA was validated using a $R D 29 B:: L U C$ reporter assay ${ }^{35}$ (Supplementary Fig. 13a). Immunoprecipitated proteins were tested in an in vitro SnRK1 $\alpha 1$ kinase assay using a similarly generated SnRK1 upstream kinase $\left(\right.$ SnAK2, ref. $\left.{ }^{36}\right)$. While incubation of recombinant SnRK1 $\alpha 1$ with immunoprecipitated SnAK2 resulted in a strong induction of SnRK1 activity, no effect was observed for the ABA-activated

Fig. 2 | SnRK2s interact with SnRK1 in a PP2CA-dependent manner. a,b, SnRK1 1 and SnRK2.2 interact in planta and the interaction is reduced over twofold in ABA. Seedlings expressing proSnRK1 $\alpha 1:: S n R K 1 \alpha 1$-GFP (a) or proSnRK2.2::SnRK2.2-GFP (b) were mock- or ABA-treated, GFP-tagged proteins were immunoprecipitated from roots and coimmunoprecipitation of SnRK2 and SnRK1 1, respectively, and this was assessed by immunodetection with the indicated antibodies. Graphs correspond to the average of four independent experiments (error bars indicate s.e.m.). $P$ values denote statistically significant differences (two-tailed Student $t$-test in a and two-tailed Welch $t$-test in b). c,d, PP2CA coimmunoprecipitates with SnRK1 $\alpha 1$-GFP (c) and SnRK2.2-GFP (d) and, proportionally to the total PP2CA levels, both interactions are reduced in ABA. Seedlings expressing proSnRK1 $\alpha$ ::SnRK1 $\alpha 1-G F P$ or proSnRK2.2::SnRK2.2-GFP were mock- or ABA-treated, GFP-tagged proteins were immunoprecipitated from roots and copurifying proteins were analysed by immunoblotting with specific antibodies. Arrows point to the bands corresponding to the expected molecular weight for PP2CA. Two independent experiments were performed with similar results (c,d). e, Bimolecular fluorescence complementation experiments show that SnRK1 $\alpha 1$ and SnRK2.2 interact only in the presence of PP2CA, and this interaction occurs mostly in the nucleus. Left panels, representative pictures of $N$. benthamiana epidermal cells expressing YFPN-SnRK1 $\alpha 1$ and YFPC-SnRK2.2 with a nuclear localized RFP (mRFP-NLS) or with PP2CA-RFP. Right panels, quantification of RFP and YFP signals (error bars indicate s.e.m.; mRFP-NLS samples, $n=9$; PP2CA-RFP samples, $n=14$ ). Scale bars, $30 \mu m$. Two independent experiments were performed with similar results. 
a
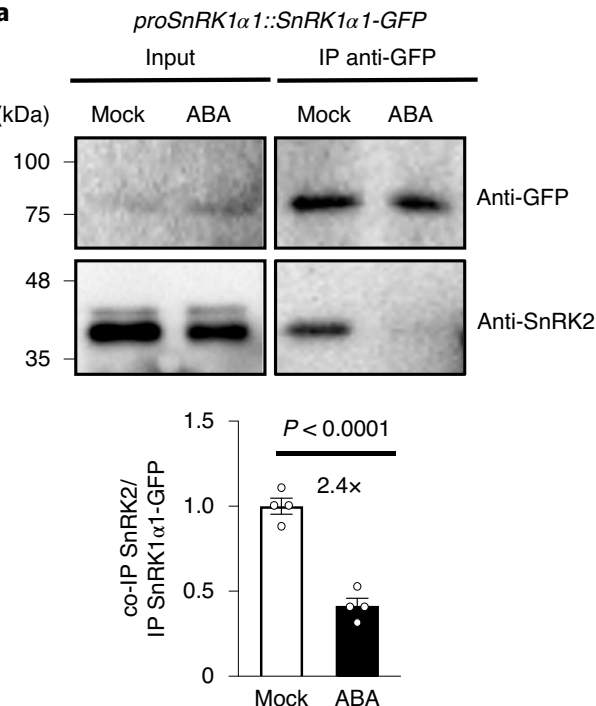

C
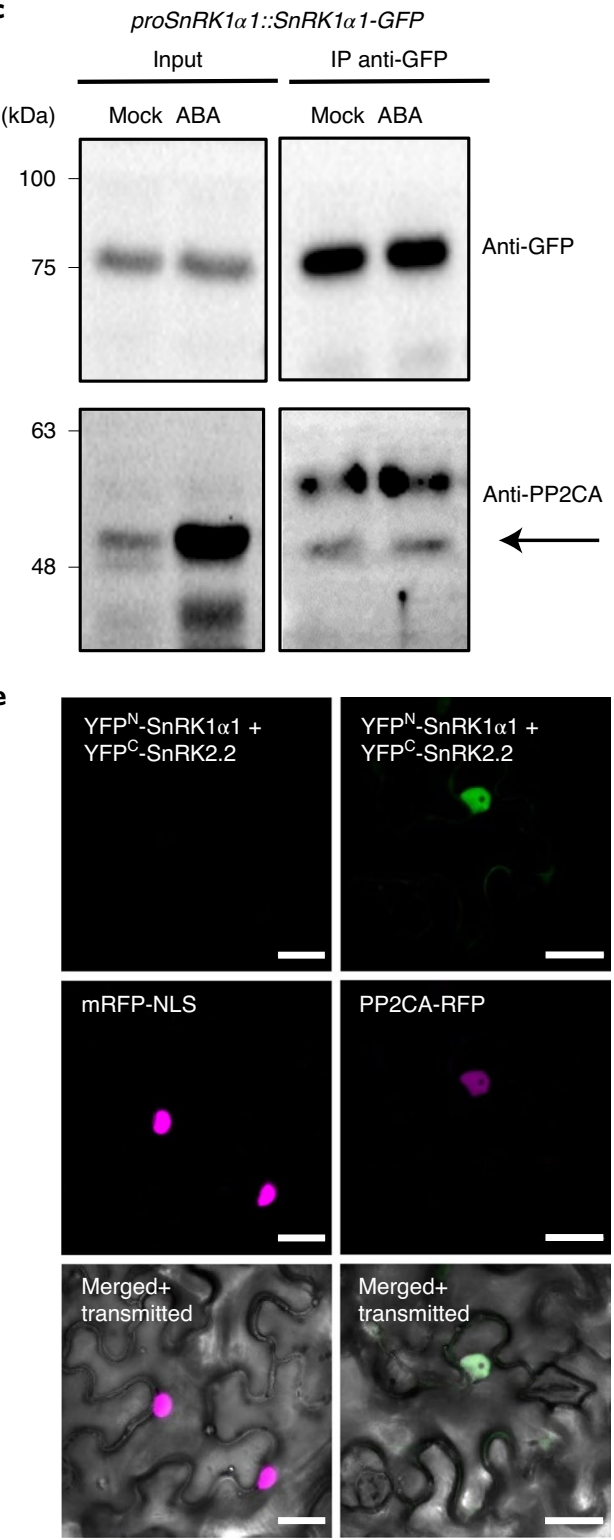

b
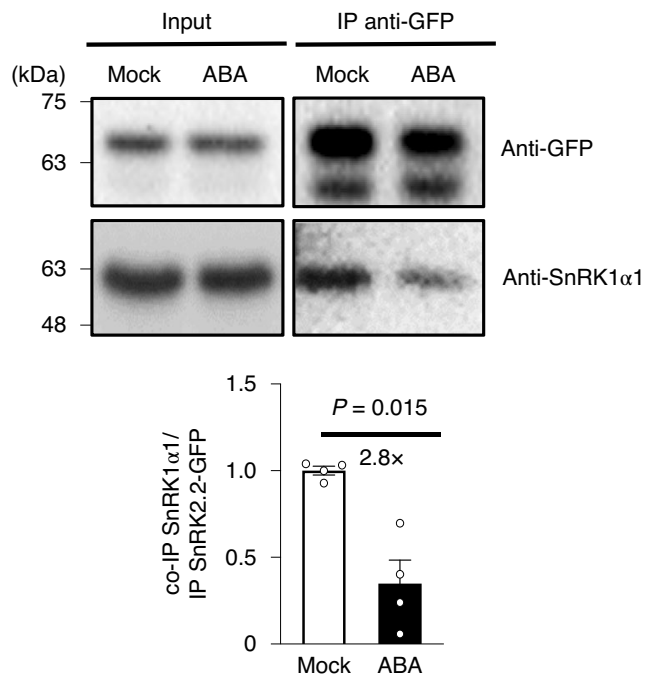

d
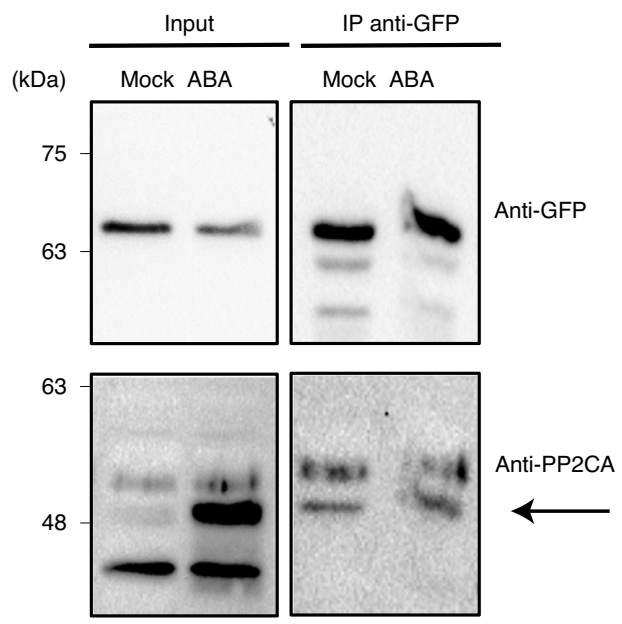
RFP signal
$\square$ YFP signal
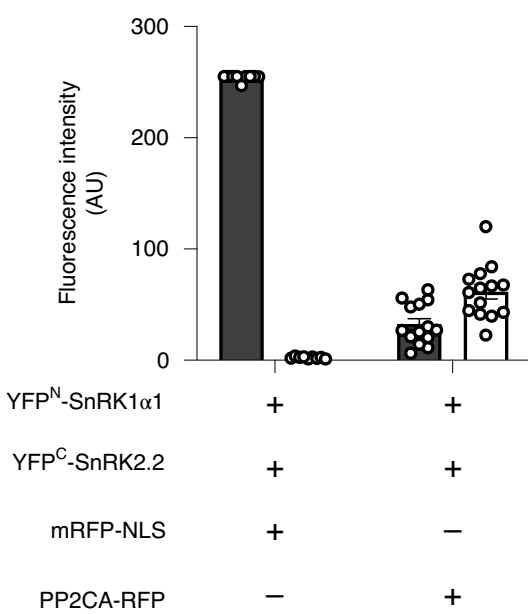
SnRK2.3, which yielded similarly low SnRK1 activities as the inactive SnRK2.3 $3^{\mathrm{K} 51 \mathrm{~N}}$ variant (Supplementary Fig. 13b,c). Altogether, these results suggest that SnRK2s promote SnRK1 signalling but this does not appear to involve direct $\operatorname{SnRK} 1 \alpha 1$ activation.

We next asked whether repression of TOR by SnRK1 always requires SnRK2s or whether this requirement is specific to ABA. To address this, we compared the inhibition of TOR by a dark-induced energy deficit in control plants, sesquia2-1 and snrk2t mutants. As expected, sesquia2-1 seedlings had a reduced capacity to repress RPS $^{\mathrm{S} 240}$ phosphorylation in response to darkness (Supplementary Fig. 14a). This is consistent with previous reports showing defective repression of TOR outputs in plants that have compromised SnRK1 signalling ${ }^{4}$. However, the snrk2t mutant displayed similar kinetics in the repression of TOR signalling as the WT (Supplementary Fig. 14b), supporting the idea that SnRK2s are only required for repressing TOR via SnRK1 in response to ABA but not energy depletion.

We noticed that, despite its $\mathrm{ABA}$ insensitivity and overall increased growth in ABA, the snrk2d mutant displayed reduced PR and LR growth in control plates compared to the WT (Fig. 3c), in accordance with a previous report ${ }^{29}$. This was fully rescued by the snrk $1 \alpha 1$ mutation, indicating that the reduced growth of the snrk $2 d$ mutant is SnRK1 $\alpha 1$-dependent and suggesting that, in the absence of $\mathrm{ABA}, \mathrm{SnRK} 2 \mathrm{~s}$ promote root growth by repressing SnRK1 $\alpha 1$ (Fig. 3c). Further supporting a growth-promoting function of SnRK2s in normal conditions, a line overexpressing SnRK2.3 had longer PR in control plates (Supplementary Fig. 15), while showing enhanced repression of $\mathrm{PR}$ growth in $\mathrm{ABA}$, in accordance with its known ABA hypersensitivity ${ }^{37}$. To assess whether the differences in growth observed in mock conditions are TOR-dependent, we grew seedlings in increasing concentrations of the TOR inhibitor
AZD8055 (AZD). The snrk2d mutant displayed a clear hyposensitivity to AZD, with differences in PR length between WT and snrk2d seedlings being strongly reduced under increasing concentrations of the inhibitor (Fig. 3d). Furthermore, a normal sensitivity to AZD was restored by the snrk $1 \alpha 1$ mutation, indicating that the lower TOR activity of the snrk2d mutant is SnRK1-dependent (Fig. 3d). To further explore how the interplay between SnRK2 and SnRK1 kinases affects TOR activity, we performed a time-course experiment to monitor the induction of RPS6 phosphorylation in response to nutrient supplementation (replacement of the growth medium with fresh medium; Fig. 3e). In WT seedlings, a marked increase in RPS6 phosphorylation was detected within the first 30 min of refreshing the medium, followed by a slight decrease and stabilization after $1 \mathrm{~h}$. In the snrk $2 d$ mutant, however, the induction of RPS6 phosphorylation was defective, but this defect was fully rescued by the snrk1 $\alpha 1$ mutation. Altogether this and the AZD sensitivity experiment show that in the snrk $2 d$ mutant TOR is repressed to a higher extent than in WT plants and that this overrepression is SnRK1-dependent. These results further suggest that in the absence of SnRK2s, basal SnRK1 activity is increased. To investigate this, we analysed WT and snrk2d seedlings with regard to the phosphorylation status of TREHALOSE PHOSPHATE SYNTHASE 5 (TPS5), an established direct target of SnRK1 (refs. ${ }^{38,39}$ ). The tps5-1 mutant is a knockout for TPS5 (ref. ${ }^{40}$ ) and served as a control for the specificity of the TPS5 antibody (Fig. 3f). We found that the levels of TPS5 phosphorylation were indeed higher in the snrk2d mutant (1.7-fold), consistent with an enhanced SnRK1 activity. To explore this further we immunoprecipitated SnRK1 $\alpha 1$ from WT and snrk2d seedlings and analysed its interaction with the SnRK1 $\beta 1$ regulatory subunit. The $\beta$-regulatory subunits are considered to act as scaffolds

Fig. 3 | SnRK2s regulate TOR and growth via SnRK1. a, The snrk1 1 1-3 mutation increases the ABA insensitivity of the snrk2d mutant during germination.

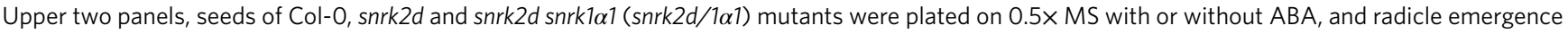
was scored at the indicated times (shown are percentages in $A B A$ as compared to the mock condition; $n=3,50$ seeds per genotype each experiment; error bars indicate s.e.m.). Different letters indicate statistically significant differences for each time point $(P<0.05$, one-way ANOVA with Tukey HSD test). Lower panel, degree of ABA insensitivity computed by normalizing the parameters scored in $A B A$ to the corresponding mock control (error bars indicate s.e.m.). $P$ values refer to the differences between snrk2d/1 $1 \alpha$ and snrk2d (one-way ANOVA with Tukey HSD test for each time point). b, The snrk1 1 1-3 mutation increases the cotyledon greening rates of the snrk2d mutant in ABA. Seeds were plated as in a and cotyledon greening was scored after $16 \mathrm{~d}$. Graph corresponds to the average of three independent experiments (100 seeds per genotype each experiment; error bars indicate s.e.m.). $P$ values denote statistically significant differences (two-tailed Student $t$-test). $\mathbf{c}$, In control conditions, the snrk2d mutant has defects in PR and LR growth that are fully rescued by the snrk1 1 mutation. In ABA, the snrk1 1 1 mutation enhances the ABA hyposensitivity of the snrk2d mutant with regard to PR length and LR density. Upper panels, representative pictures of seedlings grown vertically on $0.5 \times \mathrm{MS}$ medium for $5 \mathrm{~d}$ and transferred to $0.5 \times \mathrm{MS}$ with or without $\mathrm{ABA}$ for $8 \mathrm{~d}$. Middle panels, quantification of PR length and LR density from three independent experiments (total number of plates; WT mock, $n=21 ;$ snrk2d mock, $n=19$; snrk2d/1 $\alpha 1$ mock, $n=21$; WT ABA, $n=21$; snrk2d ABA, $n=21$; snrk2d/1 1 ABA, $n=21$. Total number of seedlings, 37-42 seedlings per genotype and condition). Upper and lower box boundaries represent the first and third quantiles, respectively, horizontal lines mark the median and whiskers mark the highest and lowest values. Lower panels, degree of ABA insensitivity computed by normalizing the parameters scored in ABA to the corresponding mock control (error bars indicate s.e.m.). Different letters indicate statistically significant differences $(P<0.05$, one-way ANOVA with Tukey HSD test). Scale bar, $1 \mathrm{~cm}$. d, The snrk2d mutant exhibits hyposensitivity to TOR inhibition by AZD8055 (AZD), and this is fully rescued by the snrk1 1 mutation. Upper panel, representative pictures of seedlings grown vertically on $0.5 \times \mathrm{MS}$ medium for $7 \mathrm{~d}$ and transferred to $0.5 \times \mathrm{MS}$ with or without the indicated AZD concentrations for $7 \mathrm{~d}$. Percentage values refer to the average increment in PR length (from the point of transfer) of the snrk2 $d$ as compared to that of the WT in each condition. Lower panel, quantification of PR length from two independent experiments (total number of plates per genotype; mock, $n=12$; $0.2 \mu \mathrm{M}$ AZD, $n=11,0.5 \mu \mathrm{M}$ AZD, $n=10$ and total number of seedlings, 20-24 per genotype and condition; error bars indicate s.e.m.). Different letters indicate statistically significant differences ( $P<0.0001$, two-way ANOVA with Tukey's HSD test). Scale bar, $1 \mathrm{~cm}$. e, The snrk2d mutant shows defective induction of TOR signalling, and this is fully rescued by the snrk1 1 mutation. Samples were collected at the indicated times following replacement of the growth medium with fresh medium. TOR activity was analysed from total protein extracts using immunoblotting and RPS6 $6^{\text {s240 }}$ phosphorylation as readout. Graph corresponds to the average of five independent experiments (error bars indicate s.e.m.). Different letters indicate statistically significant differences for each time point $(P<0.05$, one-way ANOVA with Tukey HSD test). f, The snrk2d mutant shows higher phosphorylation of TPS5, indicating higher SnRK1 activity. WT and snrk2d seedlings were grown as in c (only mock conditions). Whole seedlings were harvested and total protein extracts were analysed using Phos-tag gels to separate TPS5 phospho-proteoforms from the non-phosphorylated protein, followed by immunoblotting with a TPS5 antibody (right panel). Extracts from the tps5-1 mutant were included in regular western blot analyses (left panel) as a control for the specificity of the TPS5 antibody. All samples were run in the same gel but images were cropped for showing tps5-1 alongside WT and snrk2d. Graph corresponds to the average of three independent experiments (error bars indicate s.e.m.). g. The interaction between SnRK1 $\alpha 1$ and the SnRK1 $1 \beta 1$ regulatory subunit is enhanced in the snrk2d mutant. SnRK1 $1 \alpha 1$ was immunoprecipitated from total protein extracts of 14-d-old WT and snrk2d seedlings and copurifying proteins were analysed by immunoblotting with a SnRK1 $\beta 1$ antibody. Graph corresponds to the average of three independent experiments (error bars indicate s.e.m.). $P$ values denote statistically significant differences (two-tailed ratio $t$-test in $\mathbf{f}$ and two-tailed Student $t$-test in $\mathbf{g}$ ). 
in the SnRK1 complex, being crucial for the recruitment of specific targets $^{41}$. The SnRK1 $\beta 1$ subunit, in particular, has been implicated in the control of nitrogen and carbon metabolism ${ }^{42}$ and we therefore reasoned it could be involved in the regulation of TOR and TPS5 by the SnRK1 complex. The interaction of SnRK1 $\alpha 1$ with the SnRK1 $\beta 1$ subunit was indeed higher (1.7-fold) in the snrk2d mutant (Fig. $3 g$ ), suggesting that the lower TOR activity and increased TPS5 phosphorylation of this mutant could be the result of enhanced engagement of the SnRK1 $\beta 1$ subunit.

We conclude that SnRK2 kinases perform dual functions in plants (Fig. 4). In the absence of ABA, SnRK2s promote growth: SnRK2s are required, together with $\mathrm{PP} 2 \mathrm{Cs}$, to form 'repressor complexes' that sequester SnRK1, precluding its interaction with TOR and thereby the inhibition of TOR signalling and growth. Sequestration of SnRK $1 \alpha 1$ in these complexes is important for root growth (in the case of SnRK2.2 and SnRK2.3), and may potentially explain other reported unexpected effects of SnRK2 kinases, including SnRK2.6, in promoting metabolism, growth and development in optimal conditions $^{43,44}$. We propose that these complexes are the same as the ones performing canonical ABA signalling functions and that their disassembly requires sequestration of the PP2C repressors by the ABA-bound ABA receptors. Several lines of evidence support this. First, likewise SnRK2 $s^{45}$, the activation of SnRK1 by ABA requires relief of inhibition by PP2C phosphatases ${ }^{5}$. Second, ABA reduces the interaction of SnRK1 $\alpha 1$ with SnRK2 and PP2CA and between SnRK2 and PP2CA (Fig. 2a-d and Supplementary Fig. 10b,c). Third, SnRK1 $\alpha 1$ and SnRK2 are unable to interact in the absence of PP2Cs (Fig. 2e). Fourth, SnRK2s (SnRK2.2/SnRK2.3/SnRK2.6) are absolutely required for repressing TOR in response to $\mathrm{ABA}^{27}$ (Supplementary Fig. 7b), even though SnRK2s may be involved in TOR repression only indirectly.

In the presence of ABA, SnRK2s repress growth and this is partly accomplished by enabling SnRK1 activation by the hormone (Fig. 4): SnRK1 repressor complexes harbouring SnRK2s and PP2Cs
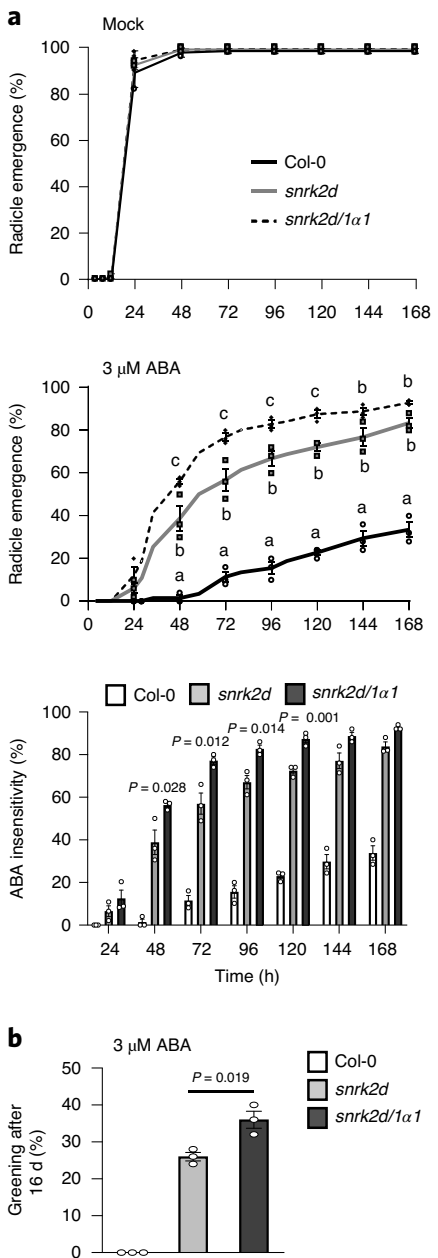

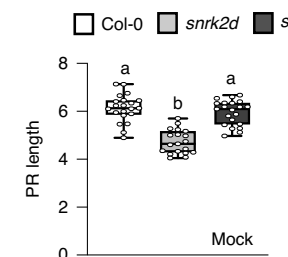

c
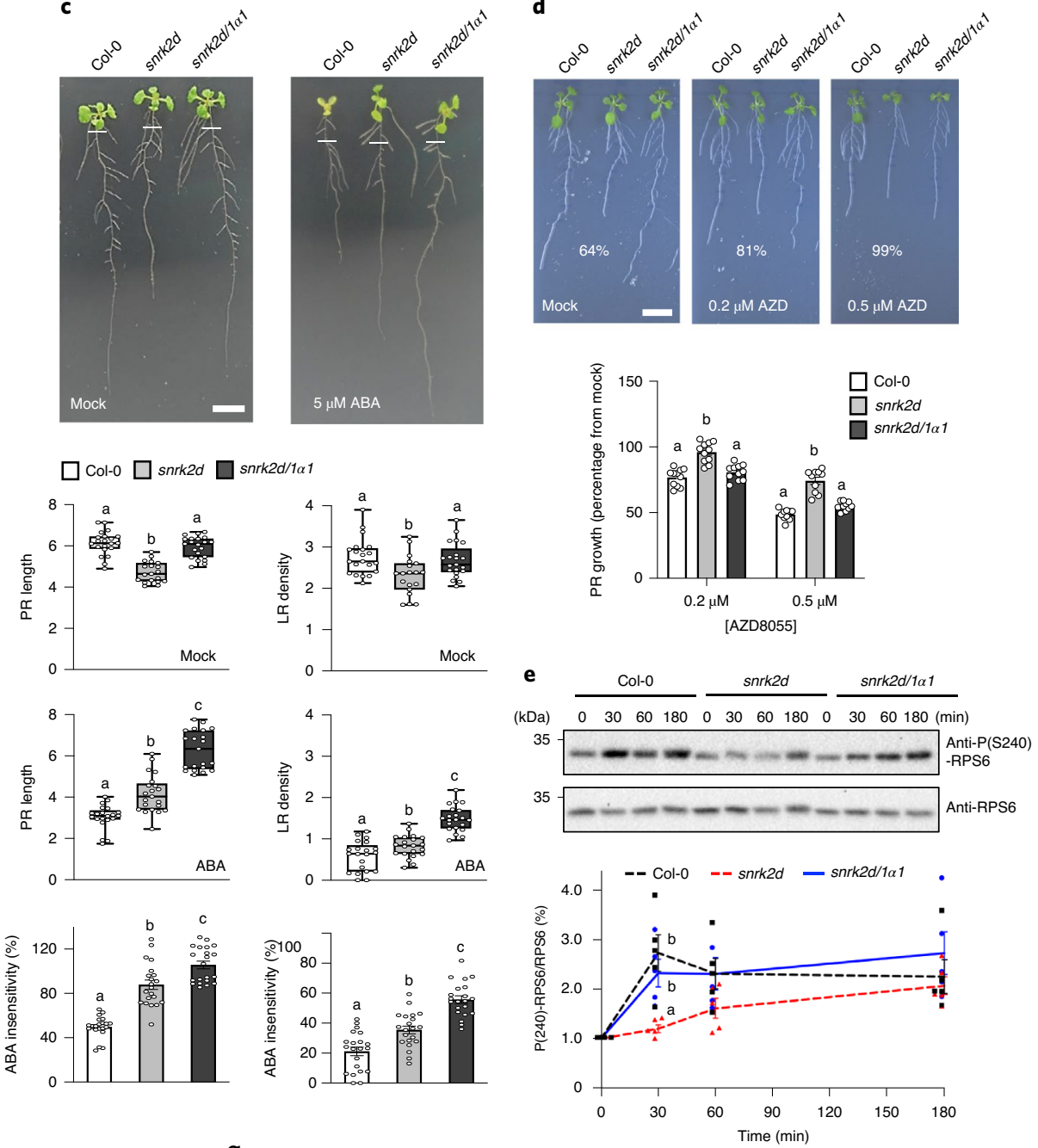

g
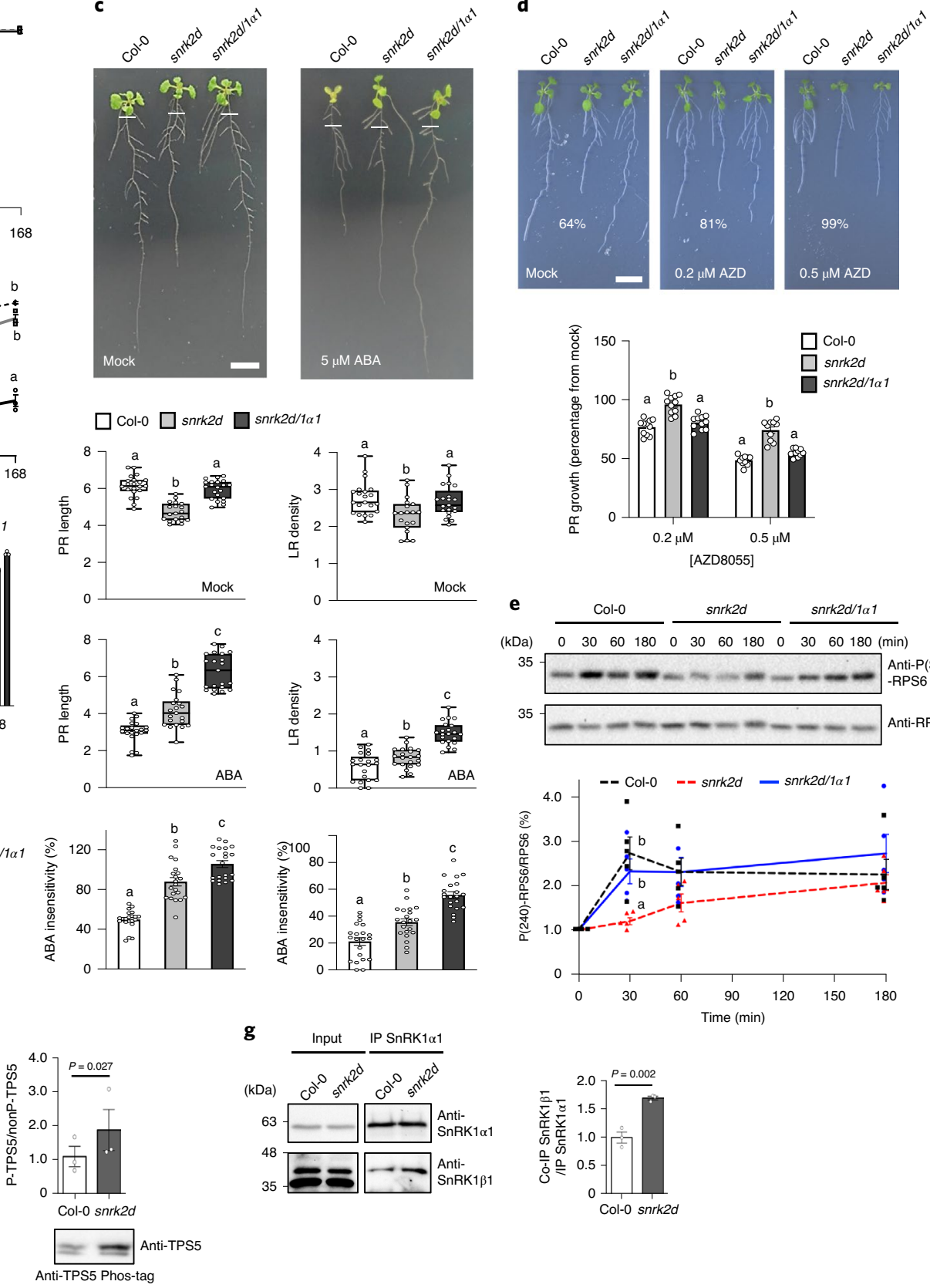


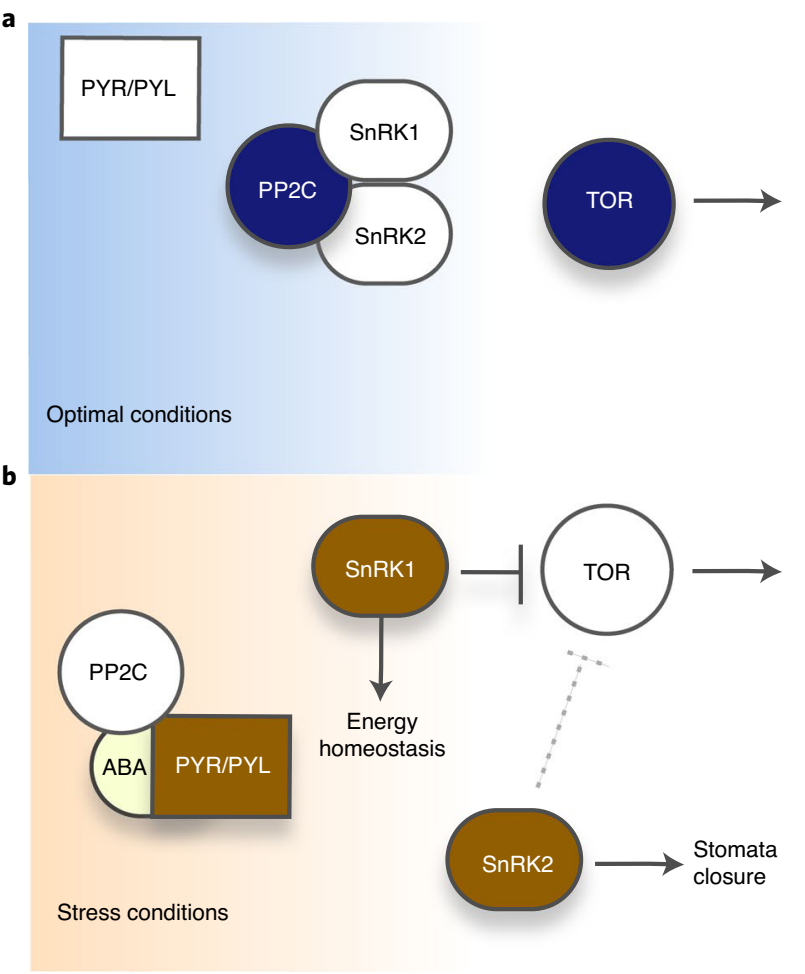

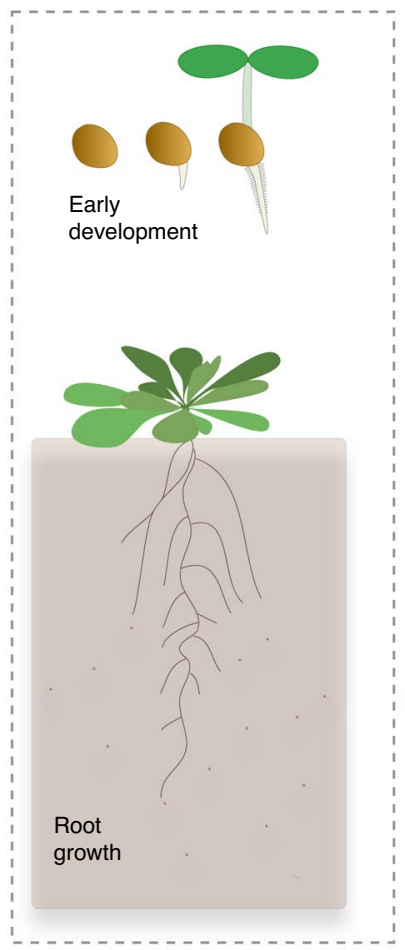

Fig. 4 | A dual function of SnRK2 kinases in the regulation of SnRK1 and growth. a, Under optimal conditions, SnRK2s promote growth. In the absence of ABA, SnRK2s are required for the formation of SnRK1 repressor complexes that also harbour PP2Cs. Sequestration of SnRK1 in these complexes is important to prevent its interaction with TOR and thereby to allow growth when conditions are favourable. b. Under stress conditions, SnRK2s inhibit growth. In the presence of ABA, SnRK2 and PP2C-containing SnRK1 repressor complexes disassemble through canonical ABA signalling involving the sequestration of PP2Cs by the ABA-bound PYR/PYL receptors. Disassembly of the complexes releases SnRK2s and SnRK1 $\alpha$ to trigger stress responses and inhibit growth. This is partly accomplished by direct TOR repression by SnRK1 but may also involve coparticipation of SnRK2 kinases. Inactive components are shown in white. Dark blue and dark orange denote components that are active under optimal conditions or under stress, respectively.

dissociate through canonical ABA signalling, releasing SnRK1 $\alpha 1$ and SnRK2 to activate stress responses. One main consequence of the ABA-triggered disassembly of these complexes is the interaction of released SnRK1 $\alpha 1$ with TOR, ultimately leading to growth inhibition. In the absence of SnRK2s these repressor complexes are not formed, rendering SnRK1 and the repression of TOR insensitive to ABA. In agreement with this, Arabidopsis raptor and lst8 mutants are $\mathrm{ABA}$ hypersensitive with regard to germination, early seedling development, and root growth ${ }^{46,47}$ while TOR overexpressors in rice display $\mathrm{ABA}$ insensitivity during germination $^{48}$. The fact that the ABA sensitivity of the sesquix2 mutants was only manifested at the level of cotyledon greening and LR density but not at the level of germination or PR length (Fig. 1), is likely to be explained by the weak nature of these mutants (Supplementary Fig. 2), by the fact that germination had to be scored from a segregating seed population and by the fact that LRs are more sensitive to $\mathrm{ABA}$ than the $\mathrm{PR}^{49}$. Repression of TOR in response to ABA may also require active input from SnRK2 (ref. ${ }^{27}$ ). However, given the lack of interaction between SnRK2s and TOR in planta (Fig. If and Supplementary Fig. 8), the simple requirement of SnRK2s to form SnRK1 repressor complexes that disassemble in response to ABA may be sufficient to explain why SnRK2s are essential for growth repression by this hormone ${ }^{27}$.

Repression of SnRK1 by SnRK2 and PP2C allows SnRK1 to be released and activated in response to ABA. However, SnRK1 is also regulated by energy depletion through mechanisms that are SnRK2-independent (Supplementary Fig. 14), suggesting that SnRK1 associates with different factors that enable its activation in response to specific signals. We propose that, in addition to its ancient and highly conserved energy-sensing function, SnRK1 evolved in land plants to respond to ABA, a crucial signal for survival in terrestrial habitats. This is accomplished through repression by the phylogenetically related subgroup III SnRK2 kinases, which belong to the same SnRK superfamily as SnRK1 (ref. ${ }^{50}$ ), but are specific to land plants ${ }^{51,52}$. Coupling the ABA-PP2C-SnRK2 module to the evolutionarily conserved SnRK1-TOR axis conferred plants the capacity to regulate growth in response to water availability and may have represented a steppingstone for the establishment of terrestrial life.

\section{Methods}

A list of all primers, antibodies and plant lines used in this study is provided in Supplementary Table 1.

Plant material and growth. All A. thaliana plants used in this study are in the Columbia (Col-0) background. Unless otherwise specified, plants were grown under long-day conditions ( $16 \mathrm{~h}$ light, $100 \mu \mathrm{mol} \mathrm{m}^{-2} \mathrm{~s}^{-1}, 22^{\circ} \mathrm{C} / 8 \mathrm{~h}$ dark, $18^{\circ} \mathrm{C}$ ) on $0.5 \times$ MS medium ( $0.05 \%$ MES and $0.8 \%$ phytoagar). The sesqui $2-1$ (snrk1 $\alpha 1-3^{-/-}$ snrk1 $\left.\alpha 2-1^{+/-}\right)$and sesqui $2-2\left(\right.$ snrk $1 \alpha 1-3^{-/-}$snrk $\left.1 \alpha 2-2^{+/-}\right)$mutants were obtained by crossing the snrk1 $\alpha 1$-3 (GABI_579E09) with the snrk1 $\alpha 2-1$ (WiscDsLox320B03) and snrk1 2 2-2 (WiscDsLox384F5) mutants, respectively. sesqui 2 individuals were always preselected on BASTA-containing medium for $5 \mathrm{~d}$ together with a BASTA-resistant 35S::GFP line (referred to as $\mathrm{Col}(\mathrm{B})$ in the text), except for germination and early development assays. Triple snrk2.2/snrk2.3/snrk1 $\alpha 1-3$ mutants (referred as snrk $2 d / \alpha 1$ in the text) were obtained by crossing $\operatorname{sn} r k 1 \alpha 1-3$ to the snrk2.2/snrk2.3 double mutant (snrk2d $)^{7}$.

Phenotype assays. For assays of ABA sensitivity during germination and early seedling development, seeds were plated on $0.5 \times$ MS supplemented or not with $\mathrm{ABA}$, and radicle emergence and cotyledon greening were computed over time under a stereoscope. 
For assaying $\mathrm{ABA}$ sensitivity during root development, seedlings were grown vertically for $5 \mathrm{~d}$ in $0.5 \times$ MS (supplied with BASTA in experiments with the sesquia 2 mutant) and transferred to $0.5 \times$ MS plates supplemented or not with ABA for another $8 \mathrm{~d}$. All computed parameters relate to the region of the root that developed after transferring the seedlings to new mock or ABA plates. For LRs, only those $\geq 0.5 \mathrm{~mm}$ long were considered.

Coimmunoprecipitation experiments. Interaction of SnRKs with TOR and RAPTOR. For assessing the interaction of SnRKs with TOR and RAPTOR, seedlings (proSnRK1 $\alpha 1:: S n R K 1 \alpha 1-G F P$, proSnRK2.2::SnRK2.2-GFP and 35S::GFP) were grown on $0.5 \times \mathrm{MS}+0.5 \%$ sucrose for $14 \mathrm{~d}(7 \mathrm{~d}$ in solid medium and $7 \mathrm{~d}$ in liquid culture) and treated with $50 \mu \mathrm{M}$ ABA for $40 \mathrm{~min}$. GFP-tagged proteins were immunoprecipitated from whole seedling cleared protein extracts using super-paramagnetic $\mu \mathrm{MAC}$ beads coupled to monoclonal anti-GFP antibody (Miltenyi Biotec), and coimmunoprecipitated proteins were analysed by western blotting using anti-GFP, anti-TOR, anti-RAPTOR, anti-SnRK1 $\alpha 1$ and anti-SnRK2 antibodies.

For immunoprecipitation of endogenous TOR, the anti-TOR antibody was coupled to Dynabeads Protein A (Invitrogen) before its addition to the whole seedling cleared protein extracts. Coimmunoprecipitated proteins were analysed by western blot with anti-TOR, anti-SnRK1 $\alpha 1$ and anti-SnRK2s antibodies.

Interaction of SnRK1 with SnRK2 and PP2CA. For assessing the interaction of SnRK1 with SnRK2 and PP2CA, seedlings (proSnRK1 $\alpha 1:: S n R K 1 \alpha 1-G F P$ proSnRK2.2::SnRK2.2-GFP and 35S::GFP) were grown on $0.5 \times \mathrm{MS}+0.5 \%$ sucrose for $14 \mathrm{~d}$ ( $7 \mathrm{~d}$ in solid medium and $7 \mathrm{~d}$ in liquid culture), and roots were rapidly harvested following a $3 \mathrm{~h}$ treatment with $50 \mu \mathrm{M}$ ABA. GFP-tagged proteins were immunoprecipitated from cleared protein extracts using super-paramagnetic $\mu \mathrm{MAC}$ beads coupled to monoclonal anti-GFP antibody (Miltenyi Biotec), and coimmunoprecipitated proteins were analysed by western blotting using anti-GFP, anti-SnRK1 $\alpha 1$, anti-SnRK2 and anti-PP2CA ${ }^{30}$ antibodies. When indicated, the SnRK1-SnRK2 interaction was analysed also from whole seedlings following a 40 min treatment with $50 \mu \mathrm{M}$ ABA as explained above for the interaction with TOR.

RPS6 $^{\text {S240 }}$ phosphorylation assays. Seedlings were grown on $0.5 \times$ MS $+0.5 \%$ sucrose for $12 \mathrm{~d}(6 \mathrm{~d}$ in solid medium + BASTA and $6 \mathrm{~d}$ in liquid culture) and treated with mock, $50 \mu \mathrm{M}$ ABA, $10 \mu \mathrm{M}$ torin 2 or $2 \mu \mathrm{M}$ AZD8055 during $4 \mathrm{~h}$. For the ABA time course, ABA $(50 \mu \mathrm{M})$ was added $1 \mathrm{~h}$ after the onset of the lights and samples were collected immediately (T0) or after 15, 30, 45, 60 and $240 \mathrm{~min}$. For the nutrient supplementation time course, the growth medium $(0.5 \times \mathrm{MS}+0.5 \%$ sucrose) was replaced with fresh medium $1 \mathrm{~h}$ after the onset of the lights and seedlings were immediately collected (T0) or after 30, 60 and $180 \mathrm{~min}$. For the sudden darkness experiments, samples were collected $3 \mathrm{~h}$ after the onset of the lights (T0) or after 1 or $3 \mathrm{~h}$ of incubation in the dark. Samples were analysed by western blot with antiphospho-RPS6 $6^{\mathrm{S} 240}$ and anti-RPS6 antibodies.

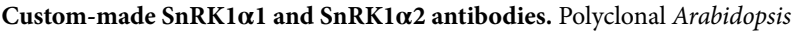
SnRK $1 \alpha 1$ and SnRK1 $\alpha 2$ antibodies were obtained by conjugating synthetic peptides (CTMEGTPRMHPAESVA and CTTDSGSNPMRTPEAGA, respectively; produced by Cocalico Biologicals, Inc.) to keyhole limpet haemocyanin and injecting two rabbits (performed by Cocalico Biologicals). Antibodies were affinity-purified using the original peptides linked to a SulfoLink matrix (Pierce) following instructions by the manufacturer.

Reporting Summary. Further information on research design is available in the Nature Research Reporting Summary linked to this article.

\section{Data availability}

All data supporting the findings of this study are available in the main text or the Supplementary Information. Additional data related to this study are available from the corresponding author upon request. All biological materials used in this study are available from the corresponding author on reasonable request. Source data are provided with this paper.

Received: 12 August 2019; Accepted: 3 September 2020; Published online: 19 October 2020

\section{References}

1. Huot, B., Yao, J., Montgomery, B. L. \& He, S. Y. Growth-defense tradeoffs in plants: a balancing act to optimize fitness. Mol. Plant 7 , 1267-1287 (2014).

2. Baena-Gonzalez, E., Rolland, F., Thevelein, J. M. \& Sheen, J. A central integrator of transcription networks in plant stress and energy signalling. Nature 448, 938-942 (2007).

3. Baena-Gonzalez, E. \& Sheen, J. Convergent energy and stress signaling. Trends Plant Sci. 13, 474-482 (2008).
4. Nukarinen, E. et al. Quantitative phosphoproteomics reveals the role of the AMPK plant ortholog SnRK1 as a metabolic master regulator under energy deprivation. Sci. Rep. 6, 31697 (2016).

5. Rodrigues, A. et al. ABI1 and PP2CA phosphatases are negative regulators of Snf1-related protein kinase1 signaling in Arabidopsis. Plant Cell 25, 3871-3884 (2013).

6. Nakashima, K., Yamaguchi-Shinozaki, K. \& Shinozaki, K. The transcriptional regulatory network in the drought response and its crosstalk in abiotic stress responses including drought, cold, and heat. Front. Plant Sci. 5, 170 (2014).

7. Fujii, H., Verslues, P. E. \& Zhu, J. K. Identification of two protein kinases required for abscisic acid regulation of seed germination, root growth, and gene expression in Arabidopsis. Plant Cell 19, 485-494 (2007).

8. Mustilli, A. C., Merlot, S., Vavasseur, A., Fenzi, F. \& Giraudat, J. Arabidopsis OST1 protein kinase mediates the regulation of stomatal aperture by abscisic acid and acts upstream of reactive oxygen species production. Plant Cell 14, 3089-3099 (2002).

9. Umezawa, T. et al. Type $2 \mathrm{C}$ protein phosphatases directly regulate abscisic acid-activated protein kinases in Arabidopsis. Proc. Natl Acad. Sci. USA 106, 17588-17593 (2009).

10. Vlad, F. et al. Protein phosphatases $2 \mathrm{C}$ regulate the activation of the Snf1-related kinase OST1 by abscisic acid in Arabidopsis. Plant Cell $\mathbf{2 1}$ 3170-3184 (2009).

11. Yoshida, R. et al. The regulatory domain of SRK2E/OST1/SnRK2.6 interacts with ABI1 and integrates abscisic acid (ABA) and osmotic stress signals controlling stomatal closure in Arabidopsis. J. Biol. Chem. 281, 5310-5318 (2006).

12. Ma, Y. et al. Regulators of PP2C phosphatase activity function as abscisic acid sensors. Science 324, 1064-1068 (2009).

13. Park, S. Y. et al. Abscisic acid inhibits type $2 \mathrm{C}$ protein phosphatases via the PYR/PYL family of START proteins. Science 324, 1068-1071 (2009).

14. Bitrian, M., Roodbarkelari, F., Horvath, M. \& Koncz, C. BAC-recombineering for studying plant gene regulation: developmental control and cellular localization of SnRK1 kinase subunits. Plant J. 65, 829-842 (2011).

15. Jossier, M. et al. SnRK1 (SNF1-related kinase 1) has a central role in sugar and ABA signalling in Arabidopsis thaliana. Plant J. 59, 316-328 (2009).

16. Lin, C. R. et al. SnRK1A-interacting negative regulators modulate the nutrient starvation signaling sensor SnRK1 in source-sink communication in cereal seedlings under abiotic stress. Plant Cell 26, 808-27 (2014).

17. $\mathrm{Lu}, \mathrm{C}$. A. et al. The SnRK1A protein kinase plays a key role in sugar signaling during germination and seedling growth of rice. Plant Cell 19, 2484-2499 (2007).

18. Radchuk, R. et al. Sucrose non-fermenting kinase 1 (SnRK1) coordinates metabolic and hormonal signals during pea cotyledon growth and differentiation. Plant J. 61, 324-338 (2010).

19. Radchuk, R., Radchuk, V., Weschke, W., Borisjuk, L. \& Weber, H. Repressing the expression of the SUCROSE NONFERMENTING-1-RELATED PROTEIN KINASE gene in pea embryo causes pleiotropic defects of maturation similar to an abscisic acid-insensitive phenotype. Plant Physiol. 140, 263-278 (2006)

20. Tsai, A. Y. \& Gazzarrini, S. AKIN10 and FUSCA3 interact to control lateral organ development and phase transitions in Arabidopsis. Plant J. 69, 809-821 (2012).

21. Tsai, A. Y. \& Gazzarrini, S. Trehalose-6-phosphate and SnRK1 kinases in plant development and signaling: the emerging picture. Front. Plant Sci. 5, 119 (2014).

22. Zhang, Y. et al. Arabidopsis sucrose non-fermenting-1-related protein kinase-1 and calcium-dependent protein kinase phosphorylate conserved target sites in ABA response element binding proteins. Ann. Appl. Biol. 153, 401-409 (2008).

23. Ramon, M. et al. Default activation and nuclear translocation of the plant cellular energy sensor SnRK1 regulate metabolic stress responses and development. Plant Cell 31, 1614-1632 (2019).

24. Lopez-Molina, L., Mongrand, S. \& Chua, N. H. A postgermination developmental arrest checkpoint is mediated by abscisic acid and requires the ABI5 transcription factor in Arabidopsis. Proc. Natl Acad. Sci. USA 98, 4782-4787 (2001)

25. Garcia, D. \& Shaw, R. J. AMPK: mechanisms of cellular energy sensing and restoration of metabolic balance. Mol. Cell 66, 789-800 (2017).

26. Dobrenel, T. et al. The Arabidopsis TOR kinase specifically regulates the expression of nuclear genes coding for plastidic ribosomal proteins and the phosphorylation of the cytosolic ribosomal protein S6. Front. Plant Sci. 7, 1611 (2016).

27. Wang, P. et al. Reciprocal regulation of the TOR kinase and ABA receptor balances plant growth and stress response. Mol. Cell 69, 100-112 e106 (2018).

28. Van Leene, J. et al. Capturing the phosphorylation and protein interaction landscape of the plant TOR kinase. Nat. Plants 5, 316-327 (2019).

29. Dietrich, D. et al. Root hydrotropism is controlled via a cortex-specific growth mechanism. Nat. Plants 3, 17057 (2017). 
30. Wu, Q. et al. Ubiquitin ligases RGLG1 and RGLG5 regulate abscisic acid signaling by controlling the turnover of phosphatase PP2CA. Plant Cell 28, 2178-2196 (2016).

31. Belin, C. et al. Identification of features regulating OST1 kinase activity and OST1 function in guard cells. Plant Physiol. 141, 1316-1327 (2006).

32. Fujii, H. \& Zhu, J. K. Arabidopsis mutant deficient in 3 abscisic acid-activated protein kinases reveals critical roles in growth, reproduction, and stress. Proc. Natl Acad. Sci. USA 106, 8380-8385 (2009).

33. Fujita, Y. et al. Three SnRK2 protein kinases are the main positive regulators of abscisic acid signaling in response to water stress in Arabidopsis. Plant Cell Physiol. 50, 2123-2132 (2009).

34. Nakashima, K. et al. Three Arabidopsis SnRK2 protein kinases, SRK2D/ SnRK2.2, SRK2E/SnRK2.6/OST1 and SRK2I/SnRK2.3, involved in ABA signaling are essential for the control of seed development and dormancy. Plant Cell Physiol. 50, 1345-1363 (2009).

35 . Fujii, $H$. et al. In vitro reconstitution of an abscisic acid signalling pathway. Nature 462, 660-664 (2009).

36. Shen, W., Reyes, M. I. \& Hanley-Bowdoin, L. Arabidopsis protein kinases GRIK1 and GRIK2 specifically activate SnRK1 by phosphorylating its activation loop. Plant Physiol. 150, 996-1005 (2009).

37. Cheng, C. et al. SCFAtPP2-B11 modulates ABA signaling by facilitating SnRK2.3 degradation in Arabidopsis thaliana. PLoS Genet. 13, e1006947 (2017).

38. Harthill, J. E. et al. Phosphorylation and 14-3-3 binding of Arabidopsis trehalose-phosphate synthase 5 in response to 2-deoxyglucose. Plant J. 47, 211-223 (2006).

39. Song, Y. et al. Identification of novel interactors and potential phosphorylation substrates of GsSnRK1 from wild soybean (Glycine soja). Plant Cell Environ. 42, 145-157 (2018)

40. Wang, X., Du, Y. \& Yu, D. Trehalose phosphate synthase 5-dependent trehalose metabolism modulates basal defense responses in Arabidopsis thaliana. J. Integr. Plant Biol. 61, 509-527 (2019).

41. Broeckx, T., Hulsmans, S. \& Rolland, F. The plant energy sensor: evolutionary conservation and divergence of SnRK1 structure, regulation, and function. J. Exp. Bot. 67, 6215-6252 (2016).

42. Wang, Y. et al. AKINbeta1, a subunit of SnRK1, regulates organic acid metabolism and acts as a global modulator of genes involved in carbon, lipid, and nitrogen metabolism. J. Exp. Bot. 71, 1010-1028 (2020).

43. Yoshida, T. et al. The role of abscisic acid signaling in maintaining the metabolic balance required for Arabidopsis growth under nonstress conditions. Plant Cell 31, 84-105 (2019).

44. Zheng, Z. et al. The protein kinase SnRK2.6 mediates the regulation of sucrose metabolism and plant growth in Arabidopsis. Plant Physiol. 153 99-113 (2010)

45. Cutler, S. R., Rodriguez, P. L., Finkelstein, R. R. \& Abrams, S. R. Abscisic acid: emergence of a core signaling network. Annu Rev. Plant Biol. 61 651-679 (2010).

46. Kravchenko, A. et al. Mutations in the Arabidopsis Lst8 and Raptor genes encoding partners of the TOR complex, or inhibition of TOR activity decrease abscisic acid (ABA) synthesis. Biochem. Biophys. Res. Commun. 467, 992-997 (2015).

47. Salem, M. A., Li, Y., Wiszniewski, A. \& Giavalisco, P. Regulatory-associated protein of TOR (RAPTOR) alters the hormonal and metabolic composition of Arabidopsis seeds, controlling seed morphology, viability and germination potential. Plant J. 92, 525-545 (2017).

48. Bakshi, A. et al. Ectopic expression of Arabidopsis target of rapamycin (AtTOR) improves water-use efficiency and yield potential in rice. Sci. Rep. 7, 42835 (2017)
49. De Smet, I. et al. An abscisic acid-sensitive checkpoint in lateral root development of Arabidopsis. Plant J. 33, 543-555 (2003).

50. Hrabak, E. M. et al. The Arabidopsis CDPK-SnRK superfamily of protein kinases. Plant Physiol. 132, 666-680 (2003).

51. Hauser, F., Waadt, R. \& Schroeder, J. I. Evolution of abscisic acid synthesis and signaling mechanisms. Curr. Biol. 21, R346-R355 (2011).

52. Umezawa, T. et al. Molecular basis of the core regulatory network in ABA responses: sensing, signaling and transport. Plant Cell Physiol. 51, $1821-1839$ (2010)

\section{Acknowledgements}

We thank J.-K. Zhu for the snrk2 mutants, M. Bennett for the SnRK2.2-GFP line, C. Koncz for the SnRK1-GFP line, X. Li for the SnRK2.3-FLAG OE line, J. Schroeder for the GFP-His-FLAG and SnRK2.6-His-FLAG OE lines, C. Mackintosh for the TPS5 antibody and the Nottingham Arabidopsis stock centre for T-DNA mutant seeds. The IGC Plant Facility (Vera Nunes) is thanked for excellent plant care. This work was supported by Fundação para a Ciência e a Tecnologia through the R\&D Units UIDB/04551/2020 (GREEN-IT-Bioresources for Sustainability) and UID/MAR/04292/2019, FCT project nos. PTDC/BIA-PLA/7143/2014, LISBOA-01-0145-FEDER-028128 and PTDC/ BIA-BID/32347/2017, and FCT fellowships/contract nos. SFRH/BD/122736/2016 (M.A.), SFRH/BPD/109336/2015 (A.C.), PD/BD/150239/2019 (D.R.B.), and IF/00804/2013 (E.B.G.). Work in P.L.R.'s laboratory was funded by MCIU grant no. BIO2017-82503-R. C.M. thanks the LabEx Paris Saclay Plant Sciences-SPS (ANR-10-LABX-040-SPS) for support. B.B.P. was funded by Programa VALi+d GVA APOSTD/2017/039. This project has received funding from the European Union Horizon 2020 research and innovation programme (grant agreement no. 867426-ABA-GrowthBalanceH2020-WF-2018-2020/H2020-WF-01-2018, awarded to B.B.P.). This work is dedicated to the memory of our beloved friend and colleague Américo Rodrigues.

\section{Author contributions}

B.B.P., M.A. and C.V. designed and performed experiments, and analysed and interpreted data. L.J.F. performed and analysed the root phenotyping experiments in low light and AZD. A.C. generated and characterized molecularly the sesqui 2 mutant lines and provided strong conceptual support. D.R.B. performed protein immunoprecipitation from protoplasts and in vitro kinase assays. A.R. contributed to the general conception of the project and the initial exploratory experiments. C.M. contributed the phospho-RPS6 antibody and expertise on molecular and plant phenotype assays related to TOR activity. P.L.R. contributed tools and expertise on PP2C-SnRK2 interactions and ABA signalling, and actively supported the conceptual work. B.B.P. and E.B.G. prepared the figures and wrote the manuscript. E.B.G. conceived the project and directed and supervised all of the research. All authors read and approved the manuscript.

\section{Competing interests}

The authors declare no competing interests.

\section{Additional information}

Supplementary information is available for this paper at https://doi.org/10.1038/ s41477-020-00778-w.

Correspondence and requests for materials should be addressed to E.B.-G.

Peer review information Nature Plants thanks Xia Li and the other, anonymous, reviewer(s) for their contribution to the peer review of this work.

Reprints and permissions information is available at www.nature.com/reprints.

Publisher's note Springer Nature remains neutral with regard to jurisdictional claims in published maps and institutional affiliations.

(C) The Author(s), under exclusive licence to Springer Nature Limited 2020 


\section{Reporting Summary}

Nature Research wishes to improve the reproducibility of the work that we publish. This form provides structure for consistency and transparency in reporting. For further information on Nature Research policies, see our Editorial Policies and the Editorial Policy Checklist.

\section{Statistics}

For all statistical analyses, confirm that the following items are present in the figure legend, table legend, main text, or Methods section.

n/a Confirmed

$\square$ \.

Х $\square$ A statement on whether measurements were taken from distinct samples or whether the same sample was measured repeatedly

$\square$ The statistical test(s) used AND whether they are one- or two-sided

$\square$ Only common tests should be described solely by name; describe more complex techniques in the Methods section.

Х $\square$ A description of all covariates tested

Х $\square$ A description of any assumptions or corrections, such as tests of normality and adjustment for multiple comparisons

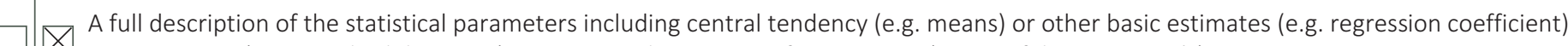

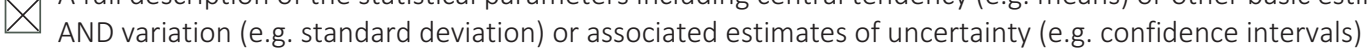

$\square$ For null hypothesis testing, the test statistic (e.g. $F, t, r$ ) with confidence intervals, effect sizes, degrees of freedom and $P$ value noted

Give $P$ values as exact values whenever suitable.

Х $\square$ For Bayesian analysis, information on the choice of priors and Markov chain Monte Carlo settings

Х $\square$ For hierarchical and complex designs, identification of the appropriate level for tests and full reporting of outcomes

\ $\square$ Estimates of effect sizes (e.g. Cohen's $d$, Pearson's $r$ ), indicating how they were calculated

Our web collection on statistics for biologists contains articles on many of the points above.

\section{Software and code}

Policy information about availability of computer code

Data collection For immunoblotting: a ChemiDoc system (Biorad) equipped with a CCD camera.

For root quantification: plates were scanned with a ScanMaker 9800 XL Microtek scanner with 1200 dpi resolution.

For confocal microscopy: Zeiss LSM 780 AxioObserver.Z1 laser-scanning microscope with C-Apochromat 40x/1.20 W corrective water immersion objective.

For qRT-PCR: Quantstudio 6 software (v1.1) on an Applied Biosystem 7900HT Fast Real-Time System.

Data analysis For quantifying band intensity in immunoblots: ImageJ (2.0.0-rc-43/1.52n; Java 1.6.0_24; 64-bit).

For quantifying roots: ImageJ.

For processing confocal microscopy images: ZEN (ZEISS Efficient Navigation) Lite 2012 imaging software (ZEN

lite 2.1; blue edition) and ImageJ.

For graph generation and statistical analyses: GraphPad Prism (v. 8.0.2) and Microsoft Excel (v16.32)

For manuscripts utilizing custom algorithms or software that are central to the research but not yet described in published literature, software must be made available to editors and reviewers. We strongly encourage code deposition in a community repository (e.g. GitHub). See the Nature Research guidelines for submitting code \& software for further information. 
Policy information about availability of data

All manuscripts must include a data availability statement. This statement should provide the following information, where applicable:

- Accession codes, unique identifiers, or web links for publicly available datasets

- A list of figures that have associated raw data

- A description of any restrictions on data availability

All data supporting the findings of this study are available in the main text or the Supplementary Information. Additional data related to this study are available from the corresponding author upon request. All biological materials used in this study are available from the corresponding author on reasonable request.

\section{Field-specific reporting}

Please select the one below that is the best fit for your research. If you are not sure, read the appropriate sections before making your selection. $\bigotimes$ Life sciences Behavioural \& social sciences Ecological, evolutionary \& environmental sciences

For a reference copy of the document with all sections, see nature.com/documents/nr-reporting-summary-flat.pdf

\section{Life sciences study design}

All studies must disclose on these points even when the disclosure is negative.

Sample size No statistical methods were used to determine sample size. Sample sizes typically used in the literature for assays on early seedling development and root architecture were also used here.

For quantification of early seedling development, 3 biologically independent experiments were performed each with 50-100 seedlings per genotype and condition.

For quantification of root parameters, a minimum of 20 seedlings (range 30-72) were used for each genotype and condition and they were grown at least as 2 independent batches (exact sample size of each experiment is given in the manuscript). Average values for all parameters were calculated for each genotype in each plate and these were used as single units for the final quantification ( $n$ therefore corresponds to the total number of plates used in each experiment).

Data exclusions For root measurements, the following criteria were pre-established for excluding specific seedlings from counts: 1) seedlings developing too much towards the edge of the plate (lateral roots could not be reliably measured in these cases). Since seedling genotypes were alternated within each plate, such exclusions did not affect systematically a particular genotype; 2 ) seedlings with roots penetrating the media (inability to clearly visualize the root and reliably determine its length); 3) seedlings clearly suffering from the transferring process (halted growth upon transfer). Seedlings meeting these criteria were excluded from Experiments of Fig. 1b, 3c, Fig. S4, Fig. S6, and Fig. S15.

Replication For all experiments a minimum of two independent biological replicates were performed and all performed replicates were included in the final data. Exceptionally the experiment shown in Supplementary Fig. 8D was not replicated as it aimed to confirm the lack of interaction between SnRK2s and TOR that had been observed in two independent experiments in the reverse direction (immunoprecipitating SnRK2 and assessing the presence of TOR).

Randomization Plant pot and plate positioning in the growth chambers was randomized to minimize positional effects during growth. Furthermore every plate contained all genotypes under comparison in alternating positions. All experiments rely on the phenotypic or molecular analyses of plants grown in the previously described manner.

Blinding

Data were always collected according to the genotype of plants.

\section{Reporting for specific materials, systems and methods}

We require information from authors about some types of materials, experimental systems and methods used in many studies. Here, indicate whether each material, system or method listed is relevant to your study. If you are not sure if a list item applies to your research, read the appropriate section before selecting a response.

Materials \& experimental systems

\begin{tabular}{l|l}
\hline$n / a$ & Involved in the study \\
\hline & $\bigotimes$ Antibodies \\
$\square$ & $\square$ Eukaryotic cell lines \\
$\square$ & $\square$ Palaeontology and archaeology \\
$\square$ & $\square$ Animals and other organisms \\
$\square$ & $\square$ Clinical data \\
& $\square$ Dual use research of concern
\end{tabular}

\begin{tabular}{l|l}
\multicolumn{2}{l}{ Methods } \\
\hline n/a & Involved in the study \\
$\bigotimes$ & $\square$ ChIP-seq \\
$\bigotimes$ & $\square$ Flow cytometry \\
$\bigotimes$ & $\square$ MRI-based neuroimaging
\end{tabular}


2. Anti-SnRK1 $\alpha 2$ polyclonal antibody from rabbit custom-made from this study (dilution 1:4000). Cocalico Biologicals Inc.

3. Anti-phospho-RPS6 (S240) polyclonal antibody from rabbit custom-made from C. Meyer (dilution 1:5000; Dobrenel et al. 2016 Front Plant Sci).

4. Anti-PP2CA polyclonal antibody from rabbit custom-made from P. Rodriguez (dilution 1:2000; Wu et al. 2016 Plant Cell).

5. Anti-SnRK2s polyclonal antibody from rabbit (Anti-SnRK2.2, SnRK2.3, SnRK2.6 | Ser/Thr-protein kinase SnRK antibodies from Agrisera, product number AS142783, species reactivity Arabidopsis thaliana, dilution 1:3000).

6. Anti-TOR polyclonal antibody from rabbit (TOR (N) antibody from Abiocode, product number R2854-1, species reactivity Arabidopsis thaliana, dilution 1:1000).

7. Anti-RPS6 monoclonal antibody from mouse (S6 Ribosomal Protein (54D2) Mouse mAb from Cell Signaling, product number 2317, species reactivity human, mouse, rat, monkey, Drosophila melanogaster, dilution 1:1000).

8. Anti-GFP monoclonal antibody from mouse (Anti-GFP from mouse $\operatorname{lgG} 1 \mathrm{k}$ (clones 7.1 and 13.1) from Roche, product number 11814460001, dilution 1:1000).

9. Anti-GFP polyclonal antibody from rabbit (GFP Rabbit IgG Polyclonal Antibody Fraction from Molecular Probes ${ }^{\circledR}$, Invitrogen, product number A-11122, dilution 1:10000).

10. Anti-HA high affinity monoclonal antibody from rat lgG1 (from Roche/Sigma, product number 11867423001, dilution 1:1000).

11. Anti-phospho-AMPKa (T172) monoclonal antibody from rabbit (Phospho-AMPKa (Thr172) (40H9) Rabbit mAb from Cell

Signaling, product number 2535, species reactivity human, mouse, rat, monkey, Drosophila melanogaster, hamster, Saccharomyces cerevisiae dilution 1:1000).

12. Anti-RAPTOR monoclonal antibody from mouse (Raptor (A-2) antibody from Santa Cruz Biotechnology, Inc., product number sc-518004, dilution 1:1000).

13. Anti-FLAG monoclonal antibody from mouse (Monoclonal ANTI-FLAG ${ }^{\oplus}$ M2 antibody from Sigma, product number F1804, dilution 1:1000)

14. Anti-SnRK1a1 commercial polyclonal antibody from rabbit (AKIN10 | SNF1-related protein kinase catalytic subunit alpha KIN10 from Agrisera, product number AS10 919, dilution 1:1000)

15. Anti-SnRK1B1 commercial polyclonal antibody from rabbit (AKINB1 | SNF1-related protein kinase regulatory subunit beta-1 from Agrisera, product number AS09 460, dilution 1:1000)

16. Anti-TPS5 polyclonal antibody from sheep (S174B) custom made from C. Mackintosh (dilution 1:1000; Harthill et al 2006 The Plant Journal)

17. Anti-sheep-PRX, Peroxidase AffiniPure Donkey Anti-Sheep IgG $(\mathrm{H}+\mathrm{L})$, Secondary antibody from Jackson ImmunoResearch Lab, inc, product number 713-035-147, dilution 1:20000).

18. Anti-mouse-PRX, Peroxidase AffiniPure goat Anti-mouse IgG $(H+L)$, Secondary antibody from Jackson ImmunoResearch Lab, inc, product number 115035146, dilution 1:20000).

19. Anti-rabbit-PRX, Peroxidase AffiniPure goat Anti-rabbit IgG $(H+L)$, Secondary antibody from Jackson ImmunoResearch Lab, inc, product number 111035144, dilution 1:20000)

1. Anti-SnRK1 $\alpha 1$ and anti-SnRK1 $\alpha 2$ antibodies were validated for A. thaliana in this study (see also Ramon et al. 2019, Plant Cell 31(7):1614-1632).

2. Anti-phospho-RPS6 (S240) antibody was previously validated for A. thaliana by C. Meyer's laboratory (https://doi.org/10.3389/ fpls.2016.01611).

3. Anti-PP2CA polyclonal antibody was previously validated for A. thaliana by P. Rodriguez's laboratory and collaborators (https:// doi.org/10.1105/tpc.16.00364, https://doi.org/10.1073/pnas.1908677116).

4. Anti-SnRK2s antibody was validated for A. thaliana by the manufacturer (https://www.agrisera.com/en/artiklar/srk-ser-thrproteinkinase-snrk.html).

5. Anti-TOR antibody was validated for A. thaliana by the manufacturer (https://www.biomol.com/products/antibodies/ primaryantibodies/general/anti-tor-n-ac-r2854-1?number=AC-R2854-1).

6. Anti-RPS6 was previously validated for A. thaliana by C. Meyer's laboratory (https://doi.org/10.3389/fpls.2016.01611).

7. Anti-GFP monoclonal antibody was validated by the manufacturer (https://www.sigmaaldrich.com/catalog/product/ roche/11814460001?lang=pt\&region=PT).

8. Anti-GFP polyclonal antibody was validated by the manufacturer (https://www.thermofisher.com/antibody/product/GFPAntibody-Polyclonal/A-11122)

9. Anti-phospho-AMPKa (T172) was previously validated for A. thaliana by J. Sheen's laboratory (DOI: 10.1038/nature06069). 10. Anti-RAPTOR antibody was validated for $\mathrm{A}$. thaliana in this study where a unique band of the expected MW was obtained. Validation in other organisms has been perfomed by the manufacturer (https://www.scbt.com/p/raptor-antibody-a-2? productCanUrl=raptor-antibody-a-2\&_requestid=1036913)

11. Anti-FLAG antibody was validated by the manufacturer (https://www.sigmaaldrich.com/catalog/product/sigma/f1804? lang=pt\&region=PT).

12. Anti-SnRK1 $\alpha 1$ and anti-SnRK1ß1 commercial antibodies were validated for A. thaliana by manufacturer (https:// www.agrisera.com/en/artiklar/akin10-snf1-related-protein-kinase-catalytic-subunit-alpha-kin10.html; https:/www.agrisera.com/en/ artiklar/akin-1.html) and Crozet et al. 2016, The Plant Journal (https://pubmed.ncbi.nlm.nih.gov/26662259/).

13. Anti-TPS5 antibody was validated in this study and previously in Harthill et al. 2006, The Plant Journal (https:// pubmed.ncbi.nlm.nih.gov/16771775/).

14. Anti-HA was validated by the manufacturer (https://www.sigmaaldrich.com/catalog/search? interface=All\&term=11867423001\&N=0\&mode=match+partialmax\&focus=product\&lang=pt\&region=global) 